\title{
The relationship between Ly $\alpha$ forest absorbers and galaxies: finding the keys at the lowest redshifts
}

\author{
John T. Stocke, Brian A. Keeney and Steven V. Penton
}

Center for Astrophysics and Space Astronomy, Department of Astrophysical and Planetary Sciences, University of Colorado, Box 389, Boulder, CO 80309, USA

\begin{abstract}
Using 138 low column density $\left(\mathrm{N}_{\mathrm{HI}}=10^{12.5-16.5} \mathrm{~cm}^{-2}\right)$ Ly $\alpha$ "forest" absorbers discovered by HST at $z \leqslant 0.15$ and nearly one million low- $z$ galaxy redshifts from on-going surveys, we find that:

1. Although nearly $80 \%$ of $\operatorname{Ly} \alpha$ forest absorbers are found within galaxy filaments, very few of them can be identified plausibly with bright $\left(\geqslant 0.1 \mathrm{~L}^{*}\right)$ galaxies. Either these absorbers are not related to any one individual galaxy or the individual galaxy to which they are related is fainter than $0.1 \mathrm{~L}^{*}\left(\mathrm{M}_{B} \geqslant-17\right)$. O VI absorbers ( $\left.\mathrm{H}+\mathrm{O} \mathrm{VI}\right)$ are found exclusively in galaxy filaments at median distances half as far away from the nearest galaxy as the Ly $\alpha$ absorber population from which they were selected $\left(\mathrm{N}_{\mathrm{HI}}=10^{13.5-16.5} \mathrm{~cm}^{-2}\right)$.

2. Our work on individual absorber-galaxy associations at the lowest redshifts finds cases where low column density $\left(\mathrm{N}_{\mathrm{HI}}=10^{13.5-16.5} \mathrm{~cm}^{-2}\right)$ metal-enriched absorbers appear to be due to unbound winds from dwarf galaxies while higher column density $\left(\mathrm{N}_{\mathrm{HI}}=10^{17.3-20} \mathrm{~cm}^{-2}\right)$ absorbers are found in the bound halos of massive galaxies. Therefore, both the statistics and a few well-studied examples lead us to propose that the low- $z$ IGM (and the Ly $\alpha$ forest absorbers in particular) are enriched in metals by outflowing winds from dwarf galaxies.
\end{abstract}

\section{Introduction}

In its very first year the Hubble Space Telescope (HST) and its UV spectrographs were used to discover that a majority of all baryons in the current day Universe are not in galaxies, but instead remain in the Intergalactic Medium (IGM). In subsequent years various HST studies have allowed an accurate "baryon census" of the IGM "warm", photoionised gas (29 $\pm 4 \%$ of all baryons; Penton et al. 2002, 2004; Papers II \& IV hereafter) as well as a first look at the "warm-hot", collisionally-ionised IGM (WHIM) in conjunction with observations made with the Far Ultraviolet Spectroscopic Explorer (FUSE). Numerical simulations (Davé et al. 2001) predict that the WHIM should contain $30-40 \%$ of all baryons at $z \approx 0$.

Because HST detected large numbers of intervening Ly $\alpha$ absorbers at $z \sim 0$ (Bahcall et al. 1991; Morris et al. 1991; Weymann et al. 1998; Impey, Petry \& Flint 1999; Papers II \& IV, and others), these discoveries have facilitated the most detailed study possible of the relationship between the various types of $\operatorname{Ly} \alpha$ absorbers and galaxies. At the highest column densities the damped-Ly $\alpha$ (DLA) systems $\left(\mathrm{N}_{\mathrm{HI}} \geqslant 10^{20.3} \mathrm{~cm}^{-2}\right)$ are identified with the gas-rich disks of galaxies spanning a large range in luminosities (0.03-1 L*) and morphologies (see the Turnshek, Rao, Rosenberg, and Zwaan contributions to this conference). Slightly weaker absorbers, the Lyman limit systems (LLS) with $\mathrm{N}_{\mathrm{HI}}=10^{17.3-20.3} \mathrm{~cm}^{-2}$, appear to be associated with the bound halos of bright $\left(\geqslant 0.3 \mathrm{~L}^{*}\right)$ galaxies (see Tripp \& Churchill contributions; Steidel 1998). But the relationship between lower column density absorbers $\left(\mathrm{N}_{\mathrm{HI}}=10^{12.5-16.5} \mathrm{~cm}^{-2}\right.$; Ly $\alpha$ forest absorbers hereafter) and galaxies is not obvious and is still controversial (see papers in 


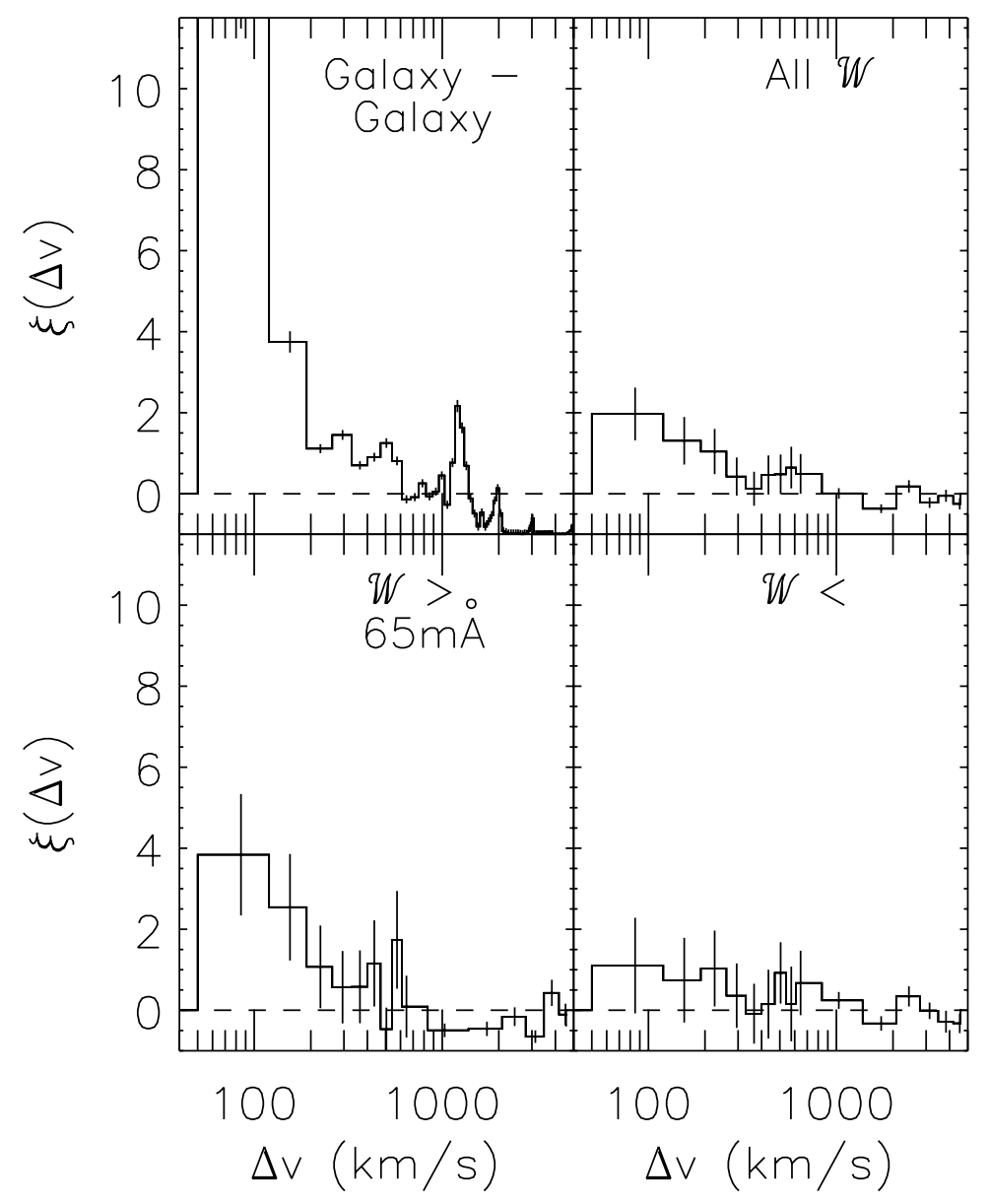

Figure 1. The Two-Point Correlation Function (TPCF) amplitude $(\xi(\Delta v))$ as a function of velocity difference $(\Delta \mathrm{v})$ for galaxies in the local Universe (top left), all $z \approx 0$ Ly $\alpha$ forest absorbers from Paper IV (top right), the stronger half of the local Ly $\alpha$ forest absorber sample (lower left) and the weaker half-sample (lower right). Notice that the galaxies and the stronger Ly $\alpha$ absorbers share the excess TPCF amplitude at $\Delta \mathrm{v} \leqslant 600 \mathrm{~km} \mathrm{~s}^{-1}$ due to super-cluster filaments and the deficit at $\Delta \mathrm{v} \geqslant 1000 \mathrm{~km} \mathrm{~s}^{-1}$ due to voids. Also notice that the TPCF amplitude for the weaker Ly $\alpha$ forest absorbers show no excess or deficit amplitude at any $\Delta \mathrm{v}$, indicating their relatively random locations in space. See Paper IV for more details.

Mulchaey \& Stocke 2002). For example, our group has previously presented evidence that most Ly $\alpha$ forest absorbers $(\sim 80 \%)$ at $z \leqslant 0.1$ are associated with galaxy filamentary structure, but not with individual galaxies. On the other hand, some weaker Ly $\alpha$ forest absorbers are found in galaxy voids (McLin et al. 2002), which proves that some Ly $\alpha$ forest absorbers are not related to galaxies at all. Clearly, much work is still to be done.

In this contribution, we present new statistical results on the relationship between various types of $\operatorname{Ly} \alpha$ forest absorbers at $z \leqslant 0.15$ and galaxies using $>910,000$ galaxy redshifts compiled using the latest data releases from various large-area redshift surveys (CfA, SDSS, 2DF/6DF, plus our own pencil-beam surveys). We also present interesting 
Table 1. Median nearest neighbour distances $\left(h_{70}^{-1} \mathrm{kpc}\right)$

\begin{tabular}{|c|c|c|c|c|c|c|c|c|}
\hline \multirow[t]{2}{*}{ Sample $^{\mathrm{a}}$} & \multicolumn{4}{|c|}{ L* REGIONS } & \multicolumn{4}{|c|}{$0.1 \mathrm{~L}^{*}$ REGIONS } \\
\hline & $\geqslant$ & $\mathrm{L}^{*}$ & & All & $\geqslant 0$ & $1 \mathrm{~L}^{*}$ & & All \\
\hline Galaxy - Galaxy & 1860 & $(500)$ & 250 & $(2000)$ & 240 & $(630)$ & 120 & $(1650)$ \\
\hline Ly $\alpha$ - Stronger Half & 1130 & $(69)$ & 450 & (69) & 450 & $(20)$ & 280 & $(20)$ \\
\hline Ly $\alpha$ - Weaker Half & 2150 & $(69)$ & 1855 & $(69)$ & 1455 & (19) & 480 & (19) \\
\hline O VI Absorbers ${ }^{\mathrm{b}}$ & 620 & (23) & 285 & (23) & 330 & (9) & 180 & (9) \\
\hline O VI Absorbers in Pairs ${ }^{c}$ & 415 & (10) & 220 & $(10)$ & - & (3) & - & (3) \\
\hline
\end{tabular}

${ }^{a}$ Numbers in parentheses are sample sizes.

${ }^{\text {b }}$ FUSE O VI absorber sample from Danforth \& Shull (2005).

c Too few absorbers in $0.1 \mathrm{~L}^{*}$ regions to report accurate median.

individual examples of absorber/galaxy associations, that illustrate the efficacy of observing QSO/galaxy pairs at the very lowest redshifts.

\section{Statistical analysis of absorber-galaxy associations}

In previous work by our group (Papers II and IV primarily), we have used a small sample (46) of $z \leqslant 0.15$ Ly $\alpha$ forest absorbers to find that higher column density Ly $\alpha$ forest absorbers cluster with each other similar to the way in which galaxies cluster (see the two-point-correlation functions (TPCF) for absorbers and galaxies in Fig. 1) and are found much closer to galaxies than weaker forest absorbers (Paper II). This led us to propose in Papers II and IV that Ly $\alpha$ forest absorbers are comprised of two populations, one found in filaments of galaxies, while the second population of statistically weaker absorbers is nearly randomly distributed in space. But, while the stronger absorbers are found in galaxy filaments, there was no clear evidence for association between individual bright galaxies and absorbers (unlike the LLS). This result plus the absence of kinematic evidence linking absorbers to their nearest galaxy neighbours (Côté et al. 2005; Putman et al. 2004) is consistent with the large-scale structure origin for Ly $\alpha$ forest absorbers found by numerical simulations (Davé et al. 1999).

Table 1 shows a summary of our new results using a sample of 138 Ly $\alpha$ forest absorbers in regions of nearby space $(z \leqslant 0.15)$ surveyed for galaxies at least to $\mathrm{L}^{*}$ (left two columns) and using 39 absorbers in regions surveyed at least to $0.1 \mathrm{~L}^{*}$ (right two columns). In order to summarise these results we present only median (i.e. typical) nearest neighbour distances for several subsets of absorbers (details to be presented in Stocke et al., in preparation). While this is the first sizable sample of forest absorbers studied for possible galaxy associations, the numbers are still modest for some of the types (sample sizes are shown in parentheses). For both the $\mathrm{L}^{*}$ and $0.1 \mathrm{~L}^{*}$ regions, the left-hand column uses only galaxies brighter than those limits while the right hand column uses all galaxies found in the regions regardless of luminosity (i.e. some galaxies fainter than those limits are included). A few results in Table 1 are noteworthy: (1) both for the $\mathrm{L}^{*}$ and $0.1 \mathrm{~L}^{*}$ complete regions the median nearest neighbour distance between all galaxies is significantly less than median Ly $\alpha$ forest absorber distances. This is the primary basis for our statements that it is difficult to ascribe absorbers to individual galaxies. We conclude that either Ly $\alpha$ forest absorbers are related to several galaxies in the filament (i.e. any gas and metals in them come from several nearby galaxies) or the individual galaxy to which any one absorber is related is typically fainter than $0.1 \mathrm{~L}^{*}$ (for which our galaxy surveying is incomplete). Work on this issue is in progress. (2) When all galaxies in these regions are included, the median distance between an O VI absorber and the nearest 
galaxy is comparable to typical distances between galaxies. Thus, it may be possible to ascribe O VI absorbers to individual galaxies, but, again, many of these galaxies must be fainter than $0.1 \mathrm{~L}^{*}$. (3) Nearest $\geqslant 0.1 \mathrm{~L}^{*}$ neighbour distances for $\mathrm{O}$ VI absorbers are typically $330 h_{70}^{-1} \mathrm{kpc}$, which indicates how far metals have been spread from bright galaxies in the present-day Universe (even if the nearest bright galaxy is not the source of the metals). We find no example of an O VI (i.e. metal enriched) absorber in a galaxy void. (4) The weaker half-sample of Ly $\alpha$ forest absorbers have nearest neighbour galaxy distances similar to those found for photoionised absorbers in numerical simulations (Davé et al. 1999); O VI absorbers are found at distances from galaxies predicted for shockheated WHIM gas (Davé et al. 1999).

Prominent in Table 1 are "O VI Absorbers in Pairs", which are found closer to galaxies than any other sample listed. These are O VI absorbers whose associated Ly $\alpha$ lines are members of line-pairs or complexes of lines with $\Delta \mathrm{v} \leqslant 200 \mathrm{~km} \mathrm{~s}^{-1}$. This radial velocity difference corresponds to a relative space velocity between absorbers which would create a post-shock temperature $\left(\mathrm{T} \sim 10^{5.5} \mathrm{~K}\right.$ ) sufficient to collisionally-ionise oxygen to $\mathrm{O}$ VI. Thus, we identify these absorbers with gas at the lower temperature end of the WHIM. Because the "O VI Absorbers in Pairs" are about half of the O VI sample discovered by Danforth \& Shull (2005), the remaining half-sample could be very diffuse photo-ionised gas at cooler temperatures and thus not WHIM. This latter speculation is controversial.

\subsection{The origin of weak metal-line systems: two examples}

Recently, our discovery of a dwarf $\left(\mathrm{M}_{B}=-14 ; \mathrm{L}=0.004 \mathrm{~L}^{*}\right)$ post-starburst galaxy $70 h_{70}^{-1} \mathrm{kpc}$ from an $\mathrm{N}_{H I} \approx 10^{16} \mathrm{~cm}^{-2}$ metal line absorber (Fig. 2 from Stocke et al. 2004) suggests an identification of $\operatorname{Ly} \alpha$ forest absorbers with low luminosity galaxies. Not only do the absorber and galaxy redshifts match to within their combined errors, but the absorber metallicity (6\% Solar) and the mean stellar metallicity of the galaxy $(\sim 10 \%)$ approximately match. Further, the absorber has an overabundance of silicon to carbon indicative of recent supernova type II enrichment. The galaxy is a pure disk system whose optical spectrum has strong Balmer absorption lines and no evidence for dust or gas (no emission lines and $\mathrm{M}_{H I} \leqslant 5 \times 10^{6} \mathrm{M}_{\odot}$; van Gorkom et al. 1993). From ratios of Lick absorption-line indices, we estimate that the mean stellar age is $3.5 \pm 1.5$ Gyrs. Taken together, this information provides a consistent picture in which a massive $\left(\geqslant 0.3 \mathrm{M}_{\odot} \mathrm{yr}^{-1}\right)$ starburst $\sim 3$ Gyrs ago created enough supernovae to blow away the remaining gas from this galaxy into the IGM. Because the dwarf is of quite low mass, this wind can easily escape from the galaxy and move to $\sim 100 h_{70}^{-1} \mathrm{kpc}$ at the required $20-30 \mathrm{~km} \mathrm{~s}^{-1}$ to create the metal-line absorber that we see between us and 3C 273 (Stocke et al. 2004). So, although the outflowing "superwind" from this galaxy cannot be traced from source to absorber, the evidence for association is considerable.

Because this is the nearest weak metal-line system known, many other similar absorbers could arise from starburst winds produced by dwarf galaxies too faint to be detected (e.g. at $z \approx 0.1$, this dwarf would have $\mathrm{m}_{B} \sim 24$ ). This explains why Lanzetta et al. (1995) find bright galaxies 100-300 $h_{70}^{-1} \mathrm{kpc}$ away from Ly $\alpha$ forest absorbers which do not have properties that correlate with absorber properties and Côté et al. (2005) find no kinematic link between bright spirals and nearby Ly $\alpha$ forest absorbers. These absorbers may have been produced not by ejecta from the bright galaxy, but by superwinds from a dwarf or dwarfs that accompany the bright galaxy. This also explains why we find a correlation between absorbers and large-scale structure, but not necessarily with individual galaxies at $\mathrm{L} \geqslant 0.1 \mathrm{~L}^{*}$ (see also Impey, Petry \& Flint 1999). Since dwarf galaxies are so numerous (1-3 $\mathrm{Mpc}^{-3}$ ), if each dwarf galaxy had at least one massive starburst that ejected most or all of its gas to a distance of $\sim 100 h_{70}^{-1} \mathrm{kpc}$, this process would be sufficient to create 


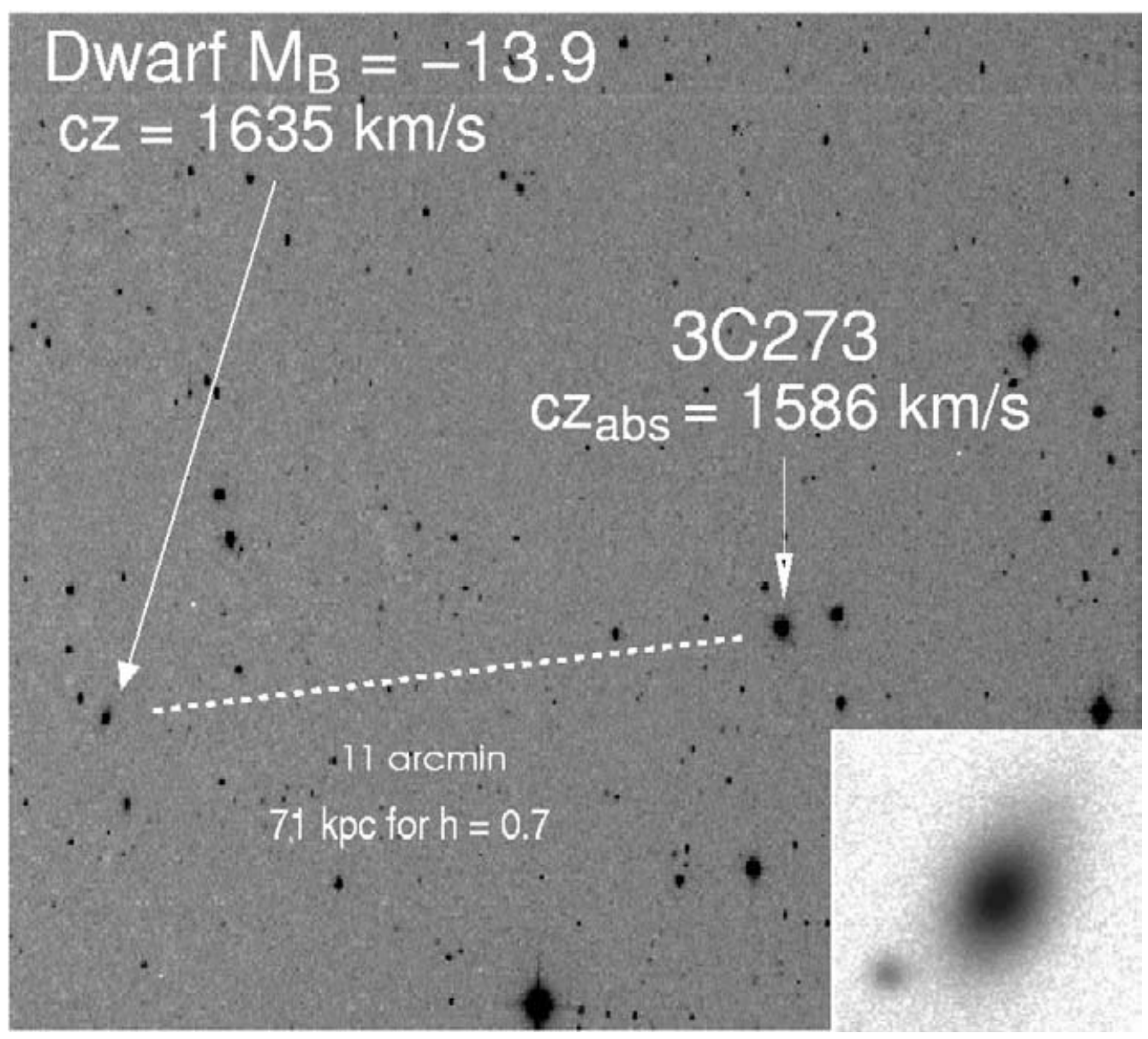

Figure 2. Digitised Sky Survey image of the region around 3C 273 (North up, East to left) showing the dwarf galaxy located $\sim 70 h_{70}^{-1} \mathrm{kpc}$ away from the absorber on the sky and at the same recession velocity to within the errors $\left( \pm 50 \mathrm{~km} \mathrm{~s}^{-1}\right)$. The R-band image of the dwarf galaxy taken with the ARC $3.5 \mathrm{~m}$ telescope at Apache Point Observatory is shown as an insert at lower right. The surface brightness profile of this galaxy is well-fitted by a pure exponential disk.

several hundred weak metal-line absorbers per unit redshift at $z \approx 2$. This number is comparable to the line density of all metal-line absorbers at $z \approx 2$. Also, while the dwarf galaxy in Fig. 2 would have been much more luminous $\left(\mathrm{M}_{B} \approx-16.5\right)$ when it was "starbursting", the present-day absence of gas means that it will no longer form stars and will eventually fade to the approximate luminosity of a Local Group dwarf spheroidal. Thus, the dwarf in Fig. 2 is the expected intermediate stage between the "faint blue galaxies" seen at $z=0.5-1$ and the present-day dwarf spheroids (Babul \& Rees 1992). That such "Cheshire Cat" galaxies might be responsible for a large portion of the Ly $\alpha$ forest was suggested by Salpeter (1993) and Charlton (1995).

Fig. 3 shows a second example of a metal-enriched wind plausibly escaping from a dwarf star-forming galaxy. In this case, the isolated dwarf starburst galaxy IC 691 is $33 h_{70}^{-1} \mathrm{kpc}$ from a QSO sight-line in which C IV is detected at a recession velocity equal to that of IC 691 to within the errors. Should the Cosmic Origins Spectrograph be placed 


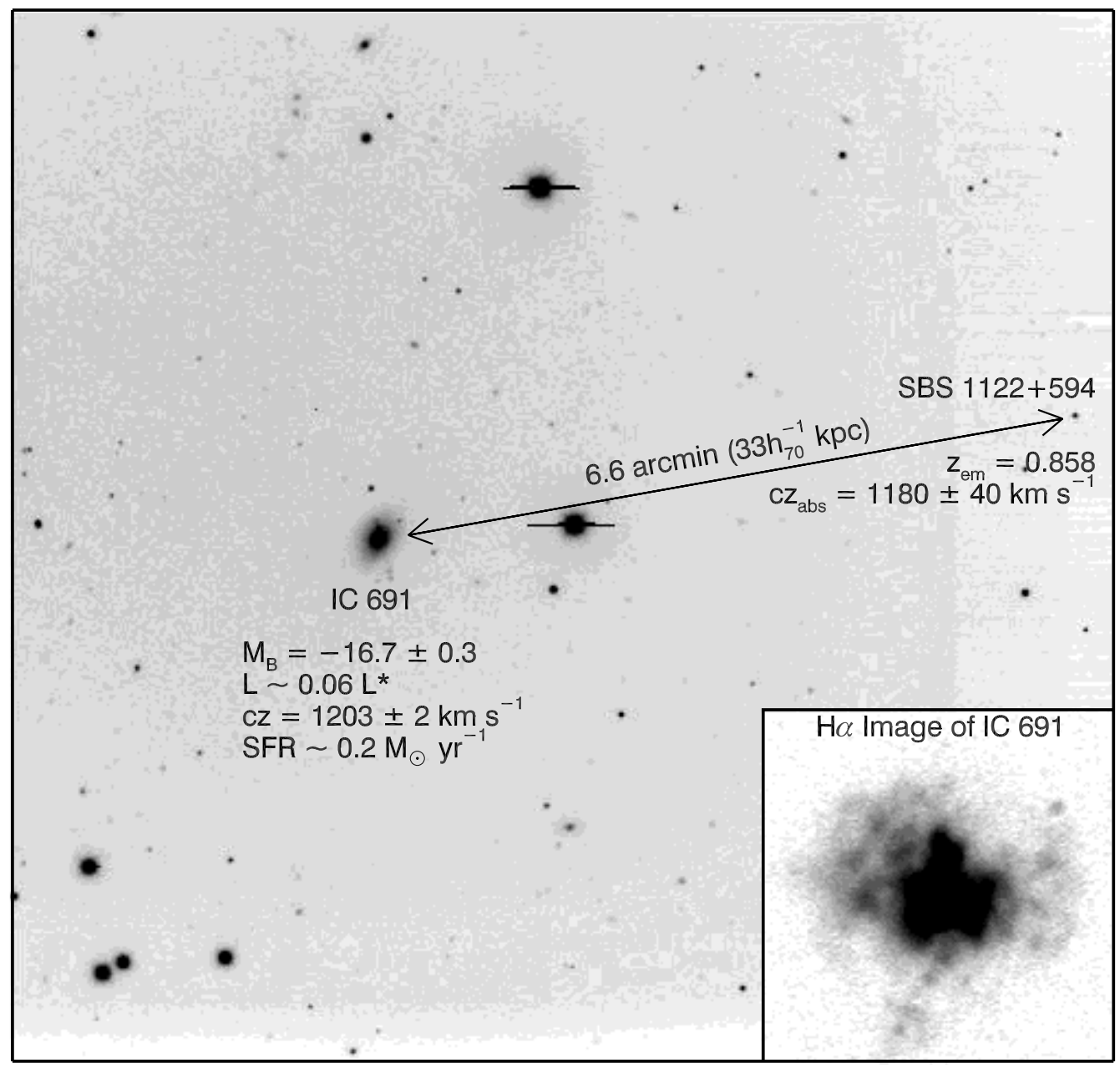

Figure 3. R-band image of the QSO/galaxy pair SBS $1122+594 /$ IC 691 , which is separated by 6.6 arc-minutes $\left(33 h_{70}^{-1} \mathrm{kpc}\right.$ projected). The inset shows a pure $\mathrm{H} \alpha$ image of IC 691 , from which we derive a star formation rate of $\sim 0.2 \mathrm{M}_{\odot} \mathrm{yr}^{-1}$. An HST/STIS spectrum of SBS $1122+594$ detects C IV absorption at a redshift of $1180 \pm 40 \mathrm{~km} \mathrm{~s}^{-1}$, comparable to that of the dwarf starburst galaxy.

into orbit, observations of many QSO/galaxy pairs will be possible enabling a detailed study of dwarf superwinds in the local Universe.

\subsection{Lyman limit systems (LLS): a "close-up view"}

But if dwarf galaxies are responsible for enriching most or all Ly $\alpha$ forest absorbers in gas, energy, and metals, what role do large galaxies like the Milky Way have in this process? Current wisdom suggests that when massive galaxies are starbursting, they eject massive superwinds into the IGM. However, at both high (Adelberger et al. 2002) and low (Heckman 2002) redshifts, wind speeds are measured against the starburst itself and so the location at which the outflow speed is measured is unknown. Thus, given an unknown amount of gravitational potential and mass loading to overcome before an outflowing wind can escape, it is not a given that this gas does, in fact, escape. Because QSOs projected near to bright galaxies sample outflows at known impact parameters, the 
ratio of wind speed to escape speed can be determined more accurately and at greater distances from the galaxy.

The QSO/galaxy pair 3C 232/NGC 3067 is a very nearby example of a LLS projected $11 h_{70}^{-1} \mathrm{kpc}$ above the plane and along the minor axis of a nearby $\left(c z=1465 \mathrm{~km} \mathrm{~s}^{-1}\right)$, nearly edge-on $0.5 \mathrm{~L}^{*}$ spiral galaxy, with a star formation rate of $1.4 \mathrm{M}_{\odot} \mathrm{yr}^{-1}$. In new work Keeney et al. (2005) use Ly $\alpha$ and H I $21 \mathrm{~cm}$ observations to obtain first measurements of the spin and kinetic temperatures of halo gas: $T_{\mathrm{s}}=435 \pm 140 \mathrm{~K}$ and $T_{\mathrm{k}} / T_{\mathrm{s}} \approx 1$. More importantly, the H I $21 \mathrm{~cm}$ emission map for the galaxy (Carilli \& van Gorkom 1992) and the H I $21 \mathrm{~cm}$ absorption for the LLS allow us to determine a space velocity $\left(\Delta \mathrm{v}=-130 \mathrm{~km} \mathrm{~s}^{-1}\right)$ for the absorber relative to the nucleus of NGC 3067; i.e. the QSO absorption line system is infalling toward the galaxy disk. Two other metal-line systems, too weak to be detected in $\mathrm{H} \mathrm{I} 21 \mathrm{~cm}$ absorption, also have kinematics that require the absorbing gas to be part of a bound halo. The H I $21 \mathrm{~cm}$ emission maps needed to determine accurate galaxy kinematics can be made only at very low redshifts $(z \leqslant 0.03)$, so inferences requiring accurate relative velocity information (e.g. infall, outflow, bound, or escaping) can be addressed directly only at very low- $z$. This argues that greater scrutiny of very nearby LLS and DLA systems should be undertaken to understand these phenomena in more detail.

A similar circumstance to NGC 3067 is present in our own Galaxy (Keeney, this conference) where outflowing gas several kpc above and below the Galactic Centre does not have sufficient velocity to escape, evidently becoming part of a bound halo and galactic "fountain". Finally, a comparison between the 3C 232/NGC 3067 LLS and Galactic high velocity clouds (like Complex C) finds similar H I column densities, galactocentric distances, kinematics, metallicities, spin temperatures, and inferred sizes.

In conclusion, based upon both a statistical study of 138 very low- $z$ Ly $\alpha$ forest absorbers and a detailed study of a few very nearby examples of QSO/galaxy pairs (three of which are presented above; see also Keeney, this conference), we suggest that lowcolumn density $\left(\mathrm{N}_{H I} \leqslant 10^{16.5} \mathrm{~cm}^{-2}\right)$ metal-line absorbers are due mostly to unbound winds from dwarf galaxies. Higher column density LLS are optically-thick, bound halos of more luminous galaxies ( $\mathrm{L}>0.3 \mathrm{~L}^{*}$ based upon the Steidel 1998 LLS survey). So, while low metallicity gas escaping from dwarf galaxies may be primarily responsible for enriching the IGM in metals and energy, it also may fall occasionally into massive galaxy halos (e.g. Complex C), stimulating star formation and solving the G dwarf problem.

We thank our collaborators J. Tumlinson, C. Danforth \& J. M. Shull for permission to use some of their work prior to publication. This work is supported by HST observing and archival grants, GO-06593, GO-09778, AR-09221, and a NASA Graduate Student Research Program (GSRP) Fellowship (NGT5-154) to B. A. K.

\section{References}

Adelberger, K. L., Steidel, C. C., Shapley, A. E., Pettini, M., 2003, ApJ, 584, 45

Babul, A., Rees, M. J., 1992, MNRAS, 255, 346

Bahcall, J. N., et al., 1991, ApJ, 377, L5

Carilli, C. L., van Gorkom, J. H., 1992, ApJ, 399, 373

Charlton, J. C., 1995, in QSO Absorption Lines, ed. G. Meylan (Berlin:Springer), p. 405

Côté, S., et al., 2005, ApJ, 618, 178

Danforth, C. D., Shull, J. M., 2005, ApJ, in press (astro-ph/0501054)

Davé, R., Hernquist, L., Katz, N., Weinberg, D. H., 1999, ApJ, 511, 521

Davé, R., et al., 2001, ApJ, 552, 473

Heckman, T. M., 2002, in ASP Conf. Ser. 254, Extragalactic Gas at Low Redshift, ed. J. S. Mulchaey \& J. T. Stocke (San Francisco: ASP), 292 
Impey, C. D., Petry, C. E., Flint, K. P., 1999, ApJ, 524, 536

Keeney, B. A., Stocke, J. T., Momjian, E., Carilli, C. L., Tumlinson, J., 2005, ApJ, 622, 267

Lanzetta, K. M., Bowen, D. V., Tytler, D., Webb, J. K., 1995, ApJ, 442, 538

McLin, K. M., Stocke, J. T., Weymann, R. J., Penton, S. V., Shull, J. M., 2002, ApJ, 574, L115

Morris, S. L., Weymann, R. J., Savage, B. D., Gilliland, R. L., 1991, ApJ, 377, L21

Mulchaey, J. S., Stocke, J. T., 2002, ASP Conf. Ser. 254, Extragalactic Gas at Low Redshift (San Francisco: ASP)

Penton, S. V., Shull, J. M., Stocke, J. T., 2000, ApJ, 544, 150 (Paper II)

Penton, S. V., Stocke, J. T., Shull, J. M., 2004, ApJS, 152, 29 (Paper IV)

Putman, M. E., Rosenberg, J. L., Ryan-Weber, E. V., Stocke, J. T., 2004, BAAS, 204, 7902

Salpeter, E. E., 1993, AJ, 106, 1265

Steidel, C. C., 1998, in ASP Conf. Ser. 136, Galactic Halos, ed. D. Zaritsky (San Francisco: ASP), 167

Stocke, J. T., Keeney, B. A., McLin, K. M., Rosenberg, J. L., Weymann, R. J., Giroux, M. L., 2004, ApJ, 609, 94

van Gorkom, J. H., Bahcall, J. N., Jannuzi, B. T., Schneider, D. P., 1993, AJ, 106, 2213

Weymann, R. J., et al., 1998, ApJ, 506, 1 